Check for updates

Cite this: RSC Adv., 2019, 9, 268

Received 2nd November 2018 Accepted 19th December 2018

DOI: $10.1039 / c 8 r a 09082 c$

rsc.li/rsc-advances

\section{Silver sulfadiazine loaded zein nanofiber mats as a novel wound dressing}

\author{
Sana Ullah, Motahira Hashmi, Muhammad Qamar Khan, Davood Kharaghani, \\ Yuseke Saito, Takayuki Yamamoto and Ick Soo Kim (D) *
}

In this report a novel antibacterial wound dressing was prepared and then characterized for required testing. We loaded silver sulfadiazine (AgSD) for the first time by electrospinning. AgSD was added in zein $(0.3 \%, 0.4 \%, 0.5 \%$, and $0.6 \%$ by weight) and was electrospun to fabricate nanofiber mats for wound dressings. Nanofiber mats were characterized by Fourier transform infrared spectroscopy (FTIR) to check if there was any chemical reaction between AgSD and zein. Morphological properties were analyzed by Scanning Electron Microscopy (SEM), which showed uniform nanofibers without any bead formation. The diameter of the nanofibers gradually decreased with an increase in the amount of AgSD, which can be associated with strong physical bonding between zein and AgSD. Thermal properties of nanofiber mats were analyzed by Thermogravimetric Analysis (TGA). X-Ray Diffraction (XRD) further demonstrated the crystalline structure of the nanofiber mats, and X-ray Photoelectron spectroscopy (XPS) was performed to confirm Ag and S contents in the prepared wound dressings. In order to investigate antibacterial properties, a disc diffusion method was employed. Bacillus and E. coli bacteria strains were used as Gram-positive and Gram-negative strains respectively. The antibacterial effectiveness of AgSD released from zein nanofibers was determined from the zone inhibition of the bacteria. The antibacterial activity of zein nanofibers loaded with drug was observed with both strains of bacteria in comparison to a control. Excellent antibacterial efficacy was attributed to the sample with $0.6 \% \mathrm{AgSD}$. Excellent release properties were also associated with the sample with $0.6 \% \mathrm{AgSD}$ in zein nanofibers. Keeping in mind the abovementioned characteristics, prepared nanofiber mats would be effective for application in wound dressings.

\section{Introduction}

Wound dressings are pieces of fabric which are used to cover a cut or a wound. They can be woven, knitted or non-woven fabric. With the increasing demand for effective wound care technology, a number of researchers are carrying out research in the field of wound care systems. Nanofiber mats have been used as wound dressings for a couple of years. It is stated that about $60 \%$ of total nanofiber production is used for biomedical purposes only. This can be for wound dressings, self-healing features, drug delivery, or implants. ${ }^{1-4}$

Silver has been among the top antibacterial drugs for decades. It has been used with a number of natural and synthetic polymers either in composite form or incorporated as nanoparticles. Silver sulfadiazine was investigated for the first time in 1974 as an effective drug for burn wound care. According to their results, sulfadiazine was not so effective for antibacterial activity in lower concentrations but they found it

Nano Fusion Technology Research Group, Institute of Frontier Fiber Engineering, Shinshu University, 3-15-1, Tokida, Ueda, Nagano, 386-8567, Japan.E-mail: kim@ shinshu-u.ac.jp; Fax: + 81-268-21-5482; Tel: + 81-268-21-5439 effective in combination with silver. ${ }^{5}$ In 1982 a study was carried out to investigate the inactivation of silver sulfadiazine by adding cerium salts. It was concluded that by the addition of cerium salt, inhibitory zone around the infected area became closer which ultimately resulted due to inactivation of silver sulfadiazine. ${ }^{6}$ A brief review was done on importance of silver nano-particles as effective antimicrobial agent. Due to availability of emerging antibacterial agents silver use was declined but it went up because of electrospinning method. In electrospinning metallic nano-particles can be used in useful manner. ${ }^{7}$ Nylon/silver composite membrane was prepared and characterized in 2010. It was observed that tensile strength of composite membrane increased as compared to nylon membranes. ${ }^{8}$ Non-woven polyvinyl alcohol (PVA), chitosan (CS), and sliver nitrate $\left(\mathrm{AgNO}_{3}\right)$ was prepared and characterized for antibacterial activity, it was concluded that prepared blends had higher antibacterial activity. ${ }^{9}$ Silver nanoparticles (AgNPs) were also added in one step electrospinning of nylon 6 , and characterized for antimicrobial activity as well as morphological and surface properties. ${ }^{\mathbf{1 0}}$

Along with above mentioned literature, AgNPs have been used in various applications incorporating with different 
natural and synthetic polymers, for example, poly methyl methacrylate (PMMA), polyvinylpyrrolidone (PVP), zein, polyacrylonitrile (PAN), chitosan, and others as well., ${ }^{411-13}$

Zein is a natural polymer which is obtained from corn. It is biodegradable and biocompatible polymer. Due to its versatile biocompatible and biodegradable properties, zein has been widely used in biomedical applications. An ultrafine membrane of zein was prepared by electrospinning and investigated the factors which affect the structure and morphology of electrospun zein nanofibers. ${ }^{14}$ Zein prolamine nanofibers were also developed and characterized for thermal and morphological properties. ${ }^{15}$ Silver and zein composites were fabricated on two different $\mathrm{pH}$ conditions and then characterized for antibacterial activity. It was concluded that silver nanoparticles were effective for antibacterial activity. ${ }^{16}$ Zein has been used effectively in biomedical applications incorporated with different antibacterial drugs i.e., cyclodextrins, eudragit, ketoprofen (KET), and ferulic acid (FA). ${ }^{17-22}$ Zein incorporation with different natural and synthetic polymers has also been used as control drug delivery and tissue engineering. ${ }^{2,4,23-25}$

Electrospinning has been used for producing nonwoven fabric since first patent for electrospinning but it became popular in $21^{\text {st }}$ century. Since then a number of researchers have been working on electrospinning of different polymers. ${ }^{26}$ In this paper, electrospinning was used to develop nanofiber mats of zein incorporated with a novel antibacterial drug silver sulfadiazine (AgSD). We are the first one to successfully load AgSD on electrospinning. AgSD has been used for wound care system in the form of hydro-gel or composite form but no one has loaded AgSD on electrospun nanofiber mats. The advantage of using this drug incorporation with natural, biocompatible and biodegradable polymer is that AgSD could occupy larger surface area. This research will be useful for industrial scale applications of AgSD loaded wound dressings.

\section{Materials \& methods}

\section{Materials}

Zein polymer was purchased from the Sigma-Aldrich Corporation (Saint Louis, MO 63103, USA), that was in powder form and contained water content less than $8 \%$. Ethanol was purchased from Sigma-Aldrich Corporation (Saint Louis, MO 63103 , USA), with purity level of $99.5 \%$. Glutaraldehyde (GA, $50 \%$ in aqueous solution) was purchased from MP Biomedical (Tokyo, Japan). Distilled water was used from laboratory. Silver(I) sulfadiazine (AgSD) was purchased from Sigma-Aldrich Corporation (Saint Louis, MO 63103, USA) in powder form with $98 \%$ purity.

\section{Method}

Zein $25 \%$ by weight was dissolved in aqueous solution (ethanol : water $=75: 25$ by weight). Solution was kept on stirring for 2 hours and then AgSD was added to the zein solution by following proportions:
Table 1 AgSD proportions in zein

\begin{tabular}{lll}
\hline Samples & Proportions by weight & Sample code \\
\hline Sample 1 & Zein 100\%, AgSD 0\% & Zein \\
Sample 2 & Zein 99.7\%, AgSD 0.3\% & $0.3 \%$ AgSD \\
Sample 3 & Zein 99.6\%, AgSD 0.4\% & $0.4 \%$ AgSD \\
Sample 4 & Zein 99.5\%, AgSD 0.5\% & $0.5 \%$ AgSD \\
Sample 5 & Zein 99.4\%, AgSD 0.6\% & $0.6 \%$ AgSD
\end{tabular}

Glutaraldehyde was added in the solution at $2.5 \mathrm{wt} \%$ for cross-linking of the solution. The prepared solution was then loaded to electrospinning to produce AgSD loaded zein nanofibers. Electrospinning was run on $15 \mathrm{kV}$ voltage, distance between needle tip and collecting roller was kept $17 \mathrm{~cm}$, and flow rate of $0.6 \mathrm{ml} \mathrm{h}^{-1}$. After the formation of nanofibers mat, it was taken off from collecting drum and was crosslinked by HCL foaming. The hydrochloric acid (HCL) foaming of zein nanofibers and zein/AgSD nanofibers was done at $30{ }^{\circ} \mathrm{C}$ for $60 \mathrm{~s}$. In this reaction, $\mathrm{GA}$ and $\mathrm{HCl}$ acted as a chemical crosslinking agent and a catalyst, respectively. This cross-linking was done to improve crystalline structure of nanofibers. Nanofibers were dried at room temperature for 3 hours and kept in air tight bags for further characterization (Table 1).

\section{Characterizations}

Bacillus and E. coli bacteria strain were used for the assessment of the antimicrobial properties by disc diffusion method. The samples were cut in round shape (disc diameter $8 \mathrm{~mm}$ ) and placed on top of plates, which cultured separately with each bacteria strain and incubated overnight (12 hours) at $37{ }^{\circ} \mathrm{C}$. The morphological properties of nanofiber mats were characterized by Scanning Electron Microscope (SEM) (JSM5300, JEOL Ltd, Japan), which was accelerated with the voltage of $12 \mathrm{kV}$. The average diameters of nanofibers were measured by taking 50 readings from random nanofibers of each sample using image analysis software (Image J, version 1.4.3). The chemical reaction between zein and AgSD were analyzed by FTIR with an ATR Prestige-21 (Shimadzu, Japan). ATR fingerprints were recorded from $400 \mathrm{~cm}^{-1}$ to $4000 \mathrm{~cm}^{-1}$. Wide angle X-ray diffractions (WAXRD) spectra were performed for the evaluation of the crystal structure at $25^{\circ} \mathrm{C}$ with nanofiber samples using a Rotaflex RT300 mA (Rigaku manufacturer, Osaka, Japan) and Nickel-filtered $\mathrm{Cu} \mathrm{K} \alpha$ radiation was used for measurements, along with an angular angle of $5 \leq 2 \theta \leq 80^{\circ}$. Thermal degradation of zein and AgSD/zein nanofibers was performed by thermogravimetric analysis using Thermo-plus TG 8120 (Rigaku Corporation, Osaka, Japan). It was operated in a static mode under air atmosphere at a heating rate of $10^{\circ} \mathrm{C} \mathrm{min}{ }^{-1}$ and a temperature range of $0-$ $500{ }^{\circ} \mathrm{C}$. The XPS analysis was examined by using a ShimadzuKratos AXIS-ULTRA HAS SV (Shimadzu, Kyoto, Japan). The release behavior of AgSD from zein nanofibers was characterized by inductively coupled plasma (ICP) atomic emission spectrometer (SHIMADZU/ICPS, 10000IV, Japan) over a period of 72 hours by immersing the nanofibers in deionized water and stirring very slowly at room temperature. 


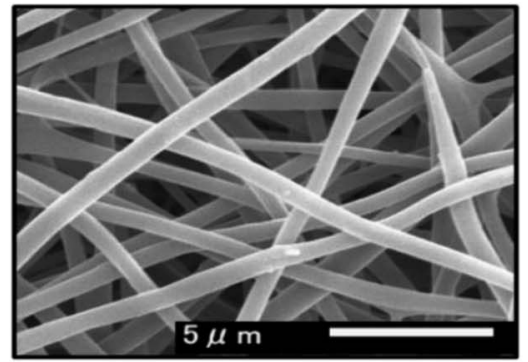

(a)

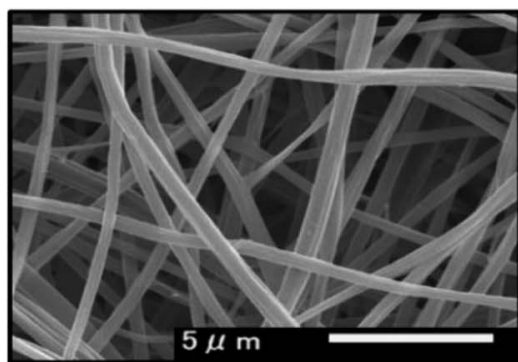

(b)

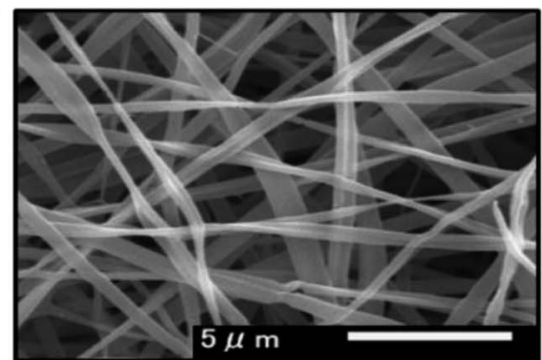

(c)

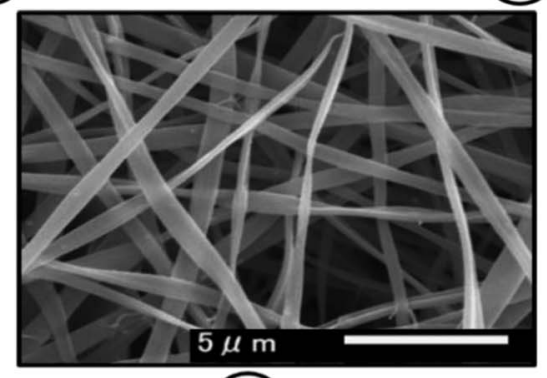

(d)

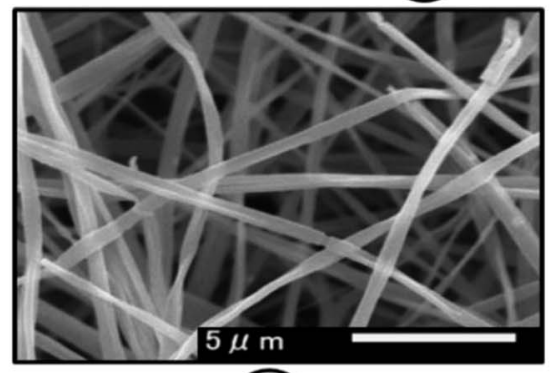

(e)

Fig. 1 SEM images of (a) pure zein (b) $0.3 \%$ AgSD in zein (c) $0.4 \%$ AgSD in zein (d) $0.5 \%$ AgSD (e) $0.6 \%$ AgSD in zein.

\section{Results and discussion}

\section{Morphological properties}

Morphological properties of prepared nanofiber mats were assessed by SEM. The SEM images in Fig. 1, showed that nanofibers of pure zein were smooth and do not contain any beads on described conditions. Addition of AgSD caused a little change in morphology as diameter is decreased and nanofiber structure was also changed. But nanofiber mats with addition of AgSD were also smooth as it can be shown in figure.

\section{Diameter interpretation}

As we mentioned above that diameter of nanofibers was changed with addition of AgSD in to the zein. Fig. 2 shows quantitative interpretation of average diameters of nanofibers. The average of 50 nanofibers from each sample showed a little change in diameters. Average diameters of zein, 0.3\% AgSD, $0.4 \% \mathrm{AgSD}, 0.5 \% \mathrm{AgSD}$, and $0.6 \% \mathrm{AgSD}$ are $236.42 \mathrm{~nm}$, $227.98 \mathrm{~nm}, 227.90 \mathrm{~nm}, 226.76 \mathrm{~nm}$, and $226.36 \mathrm{~nm}$. It was observed that all the diameters were in descending order with increase in AgSD content. Usually addition of nanoparticles or additives increase the diameter of nanofibers but in this case

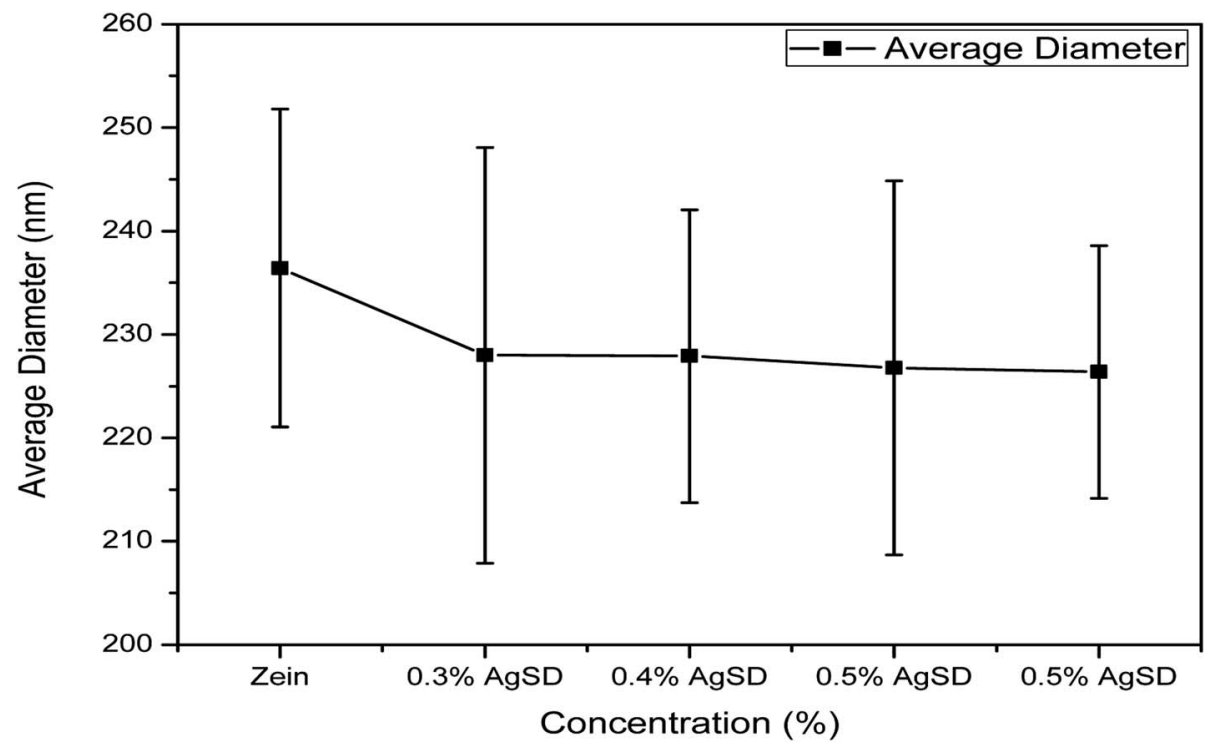

Fig. 2 Diameter distribution graph of zein and AgSD/zein nanofibers. 

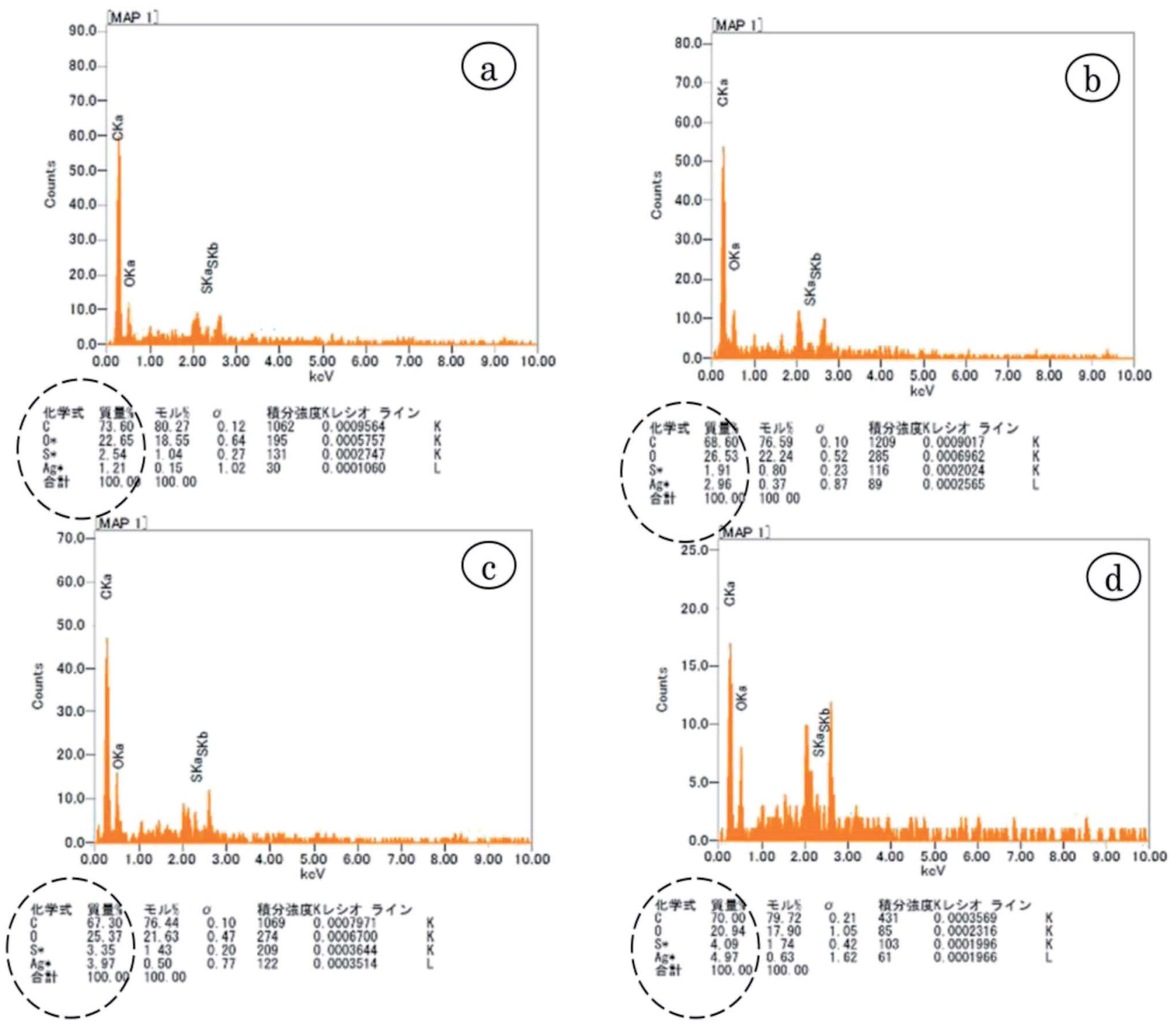

Fig. 3 EDX analysis of (a) $0.3 \%$ AgSD in zein (b) $0.4 \%$ AgSD in zein (c) $0.5 \%$ AgSD in zein (d) $0.6 \%$ AgSD in zein.

results were opposite which can be associated with packing of zein backbone due to strong physical bonding between zein and AgSD.

\section{EDX analysis}

EDX analysis was done for the quantitative measurement of Ag and sulfur content in the nanofibers. Fig. 3 shows that $\mathrm{Ag}$ and sulfur content increased as concentration of AgSD in the nanofiber increased. So, it was a direct check on the presence of $\mathrm{Ag}$ and sulfur as active ingredients of the prepared nanofibers mats. It was also observed in the EDX analysis that both Ag and $S$ showed ascending trend, as the percentage of AGSD was increased in nanofibers mat, the quantity of $\mathrm{Ag}$ and $\mathrm{S}$ also increased in EDX analysis.

\section{X-ray diffraction (XRD)}

XRD was done to confirm crystalline structure of nanofiber mats (Fig. 4). Pure zein nanofibers showed peak at $2 \theta=9^{\circ}$, and a wide peak at $2 \theta=20^{\circ}$. while zein did not exhibit any peak up to $2 \theta=80^{\circ}$. Also from literature it was confirmed that pure zein powder also exhibits the same trend which is clear evidence that zein had retained its structure in nanofiber form too. It was also observed that in pure zein, shorter peaks represented the intra-helix average backbone distance ( $\alpha$-helix structure of pure zein) while broader spacing was related intrahelix packing between the neighboring chains.

Addition of AgSD in zein presented sharp peaks at $2 \theta=28^{\circ}$, $2 \theta=31^{\circ}, 2 \theta=47^{\circ}, 2 \theta=56^{\circ}$, and $2 \theta=58^{\circ}$. Except from the two peaks $\left(2 \theta=9^{\circ}\right.$ and $\left.2 \theta=20^{\circ}\right)$, all other peaks represents the presence of AgSD and all these peaks were associated with AgSD. From XRD graph it was also observed that structural stability of zein did not changed by the addition of AgSD, although AgSD showed their peaks in nanofiber mats of zein/ $\mathrm{AgSD}$. Presence of AgSD in zein nanofibers were also proven by XPS analysis. 


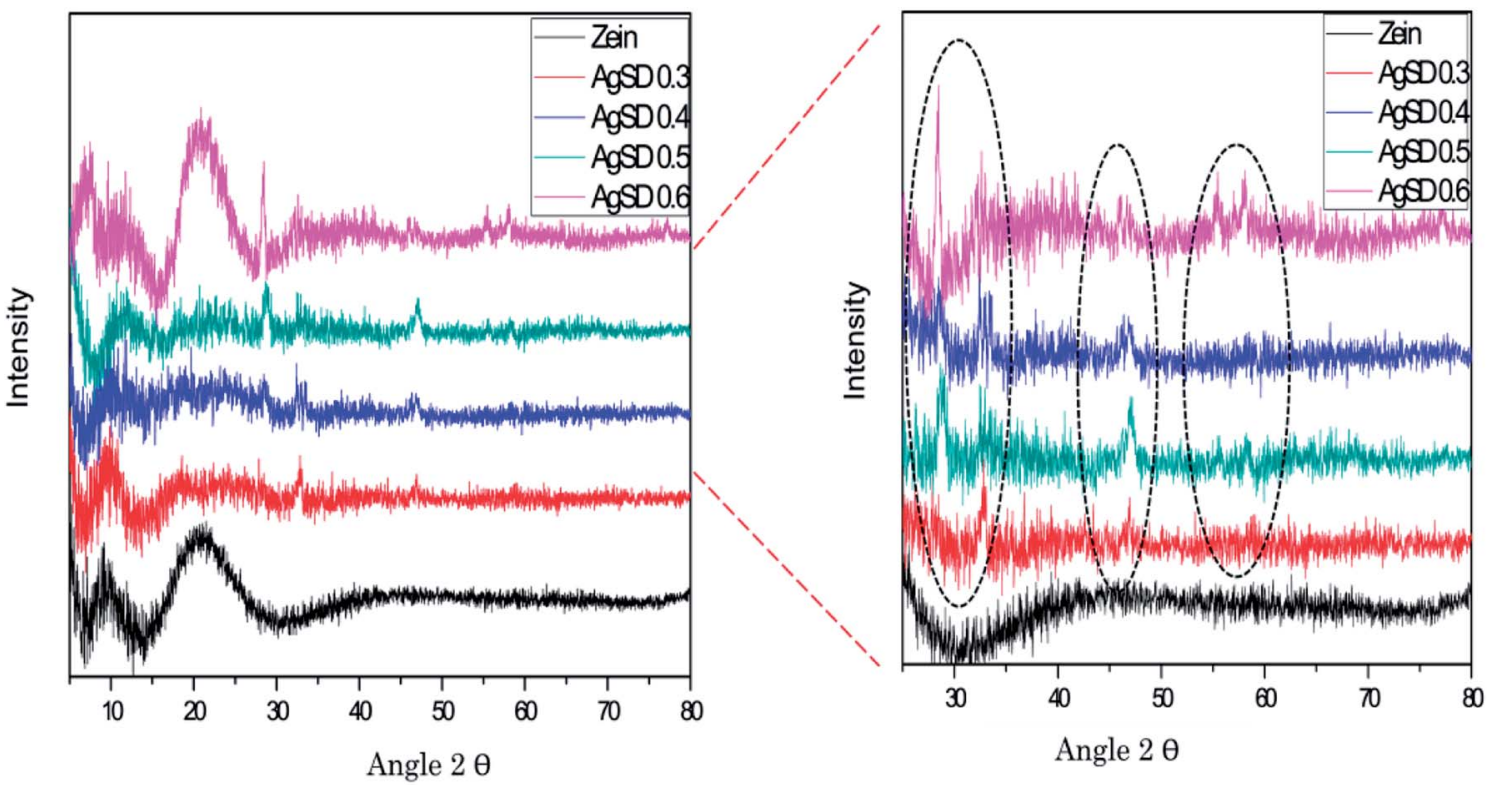

Fig. 4 X-ray diffraction analysis of zein and AgSD/zein nanofibers.

\section{X-ray photoelectron spectroscopy (XPS)}

XPS analysis was done to confirm the presence of AgSD in nanofiber mats (Fig. 5). As AgSD consists of Ag (silver) and $\mathrm{S}$ (sulfur), so XPS results for $\mathrm{Ag}(3 \mathrm{p})$ and sulfur (2p and $2 \mathrm{~s}$ ) were obtained. Ag 3p spectra had two peaks at $568.0 \mathrm{eV}\left(\mathrm{Ag} 3 \mathrm{p}_{3 / 2}\right)$ and $601.0 \mathrm{eV}\left(\mathrm{Ag} 3 \mathrm{p}_{1 / 2}\right)$ with a slit of $33 \mathrm{eV}$. Those peaks were related to metallic $\mathrm{Ag}$ in nanofibers. The standard peaks for $\mathrm{Ag}$ $3 p_{3 / 2}$ and $\mathrm{Ag} 3 \mathrm{p}_{1 / 2}$ were recorded $570.0 \mathrm{eV}$ and $604.0 \mathrm{eV}$ respectively. Spectra of $S 2 \mathrm{~s}$ had shown peak at binding energy of $230.0 \mathrm{eV}$ and spectra of S $2 \mathrm{p}$ at $163.0 \mathrm{eV}$ while standard peaks for S 2s and S 2p were recorded at $231.0 \mathrm{eV}$ and $164.5 \mathrm{eV}$. It was also observed that intensity of described peaks were higher as concentration of AgSD was increased in zein nanofibers. From all of these results it was clear evidence of silver and sulfur presence in the nanofiber mats as all above mentioned peaks are related to $\mathrm{Ag}$ and $\mathrm{S}$.
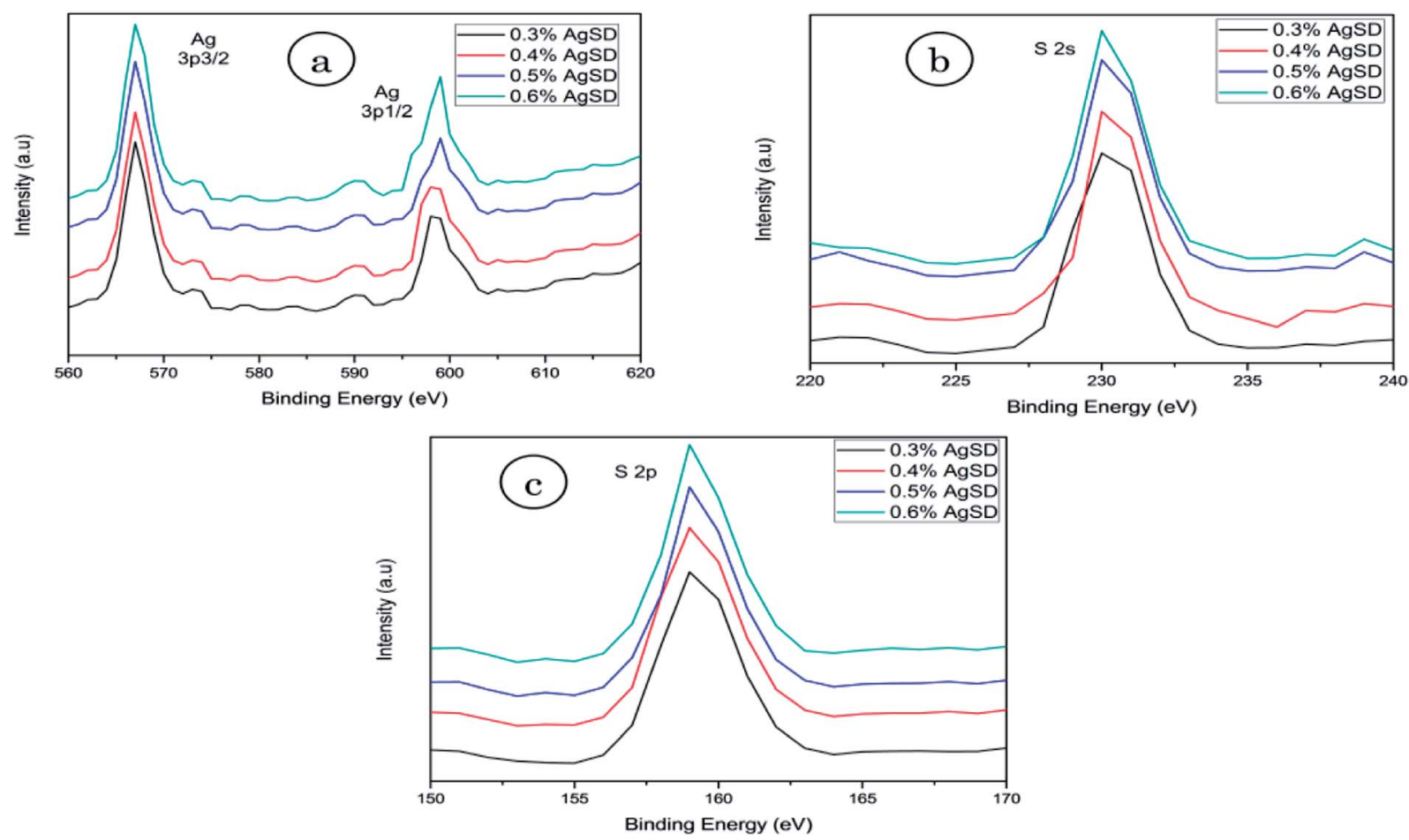

Fig. 5 XPS analysis of (a) silver $3 p$ (b) sulfur $2 s$ (c) sulfur $2 p$. 


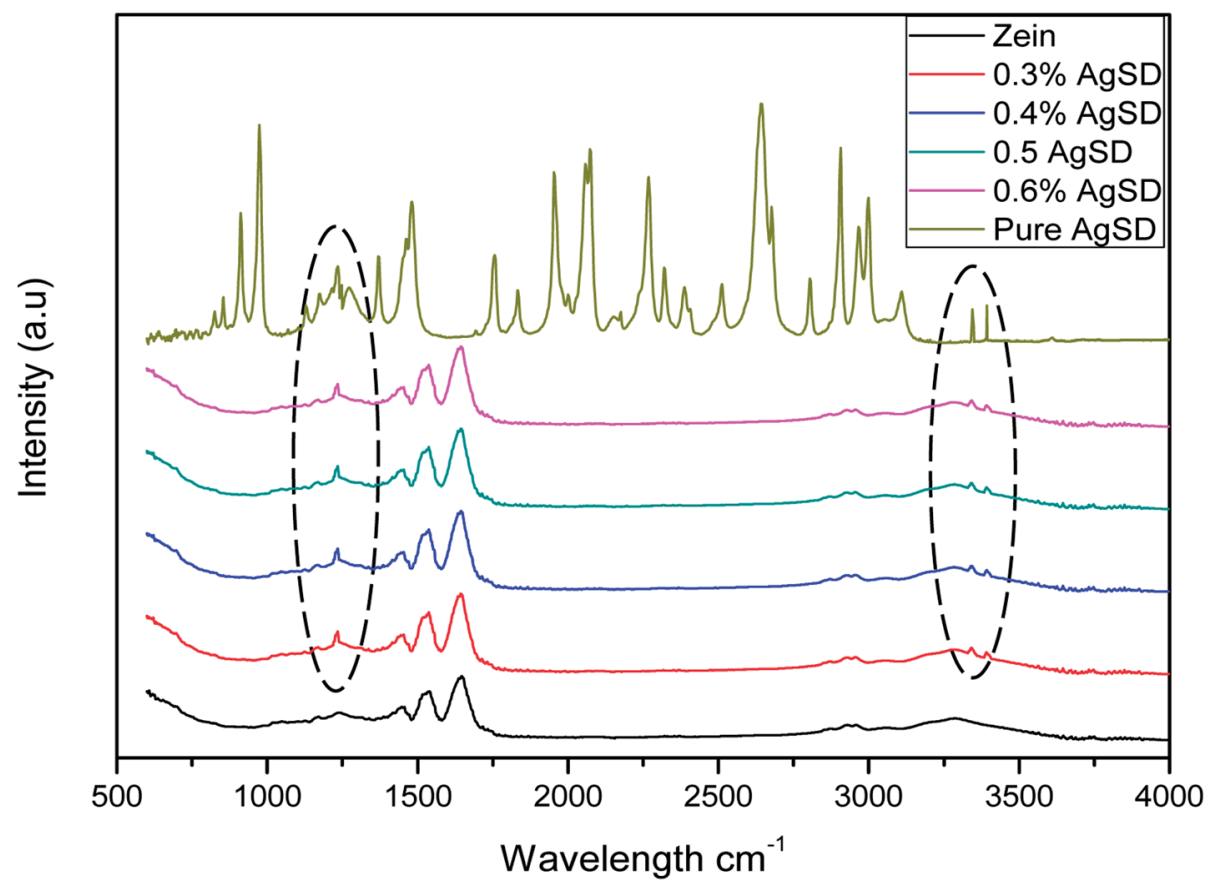

Fig. 6 FTIR fingerprints of zein and AgSD/zein nanofibers.

\section{Fourier-transform infrared spectroscopy (FTIR)}

FTIR spectra of pure zein and composite nanofibers of Ag/zein are shown in Fig. 6. It was observed that AgSD had shown a sharp peak at $1231.6 \mathrm{~cm}^{-1}$ which is associated with the main component of AgSD that is $\mathrm{SO}_{2}$ asymmetric stretching. Along with this peak, specimen containing AgSD had also shown peaks on $3341.52 \mathrm{~cm}^{-1}$ and $3391.07 \mathrm{~cm}^{-1}$ which were associated with stretching band of amine $\left(\mathrm{NH}_{2}\right)$. It was also observed that addition of AgSD did not change the spectra of pure zein, which was indication that there was no chemical reaction between zein and AgSD. ${ }^{27}$ The band corresponding to the stretching of the $\mathrm{N}-\mathrm{H}$ and $\mathrm{O}-\mathrm{H}$ bonds of the amino acids of the protein appeared between 2800 and $3500 \mathrm{~cm}^{-1}$. Another peak was observed at $1650 \mathrm{~cm}^{-1}$, which was corresponding to stretching of the carbonyl $(\mathrm{C}=\mathrm{O})$ of amide groups belonging to the peptide groups. The peak at $1540 \mathrm{~cm}^{-1}$ was associated with angular deformational vibrations of the $\mathrm{N}-\mathrm{H}$ bond, and finally, the band at $1230 \mathrm{~cm}^{-1}$ was associated with the axial deformational vibration of the $\mathrm{C}-\mathrm{N}$ bond.

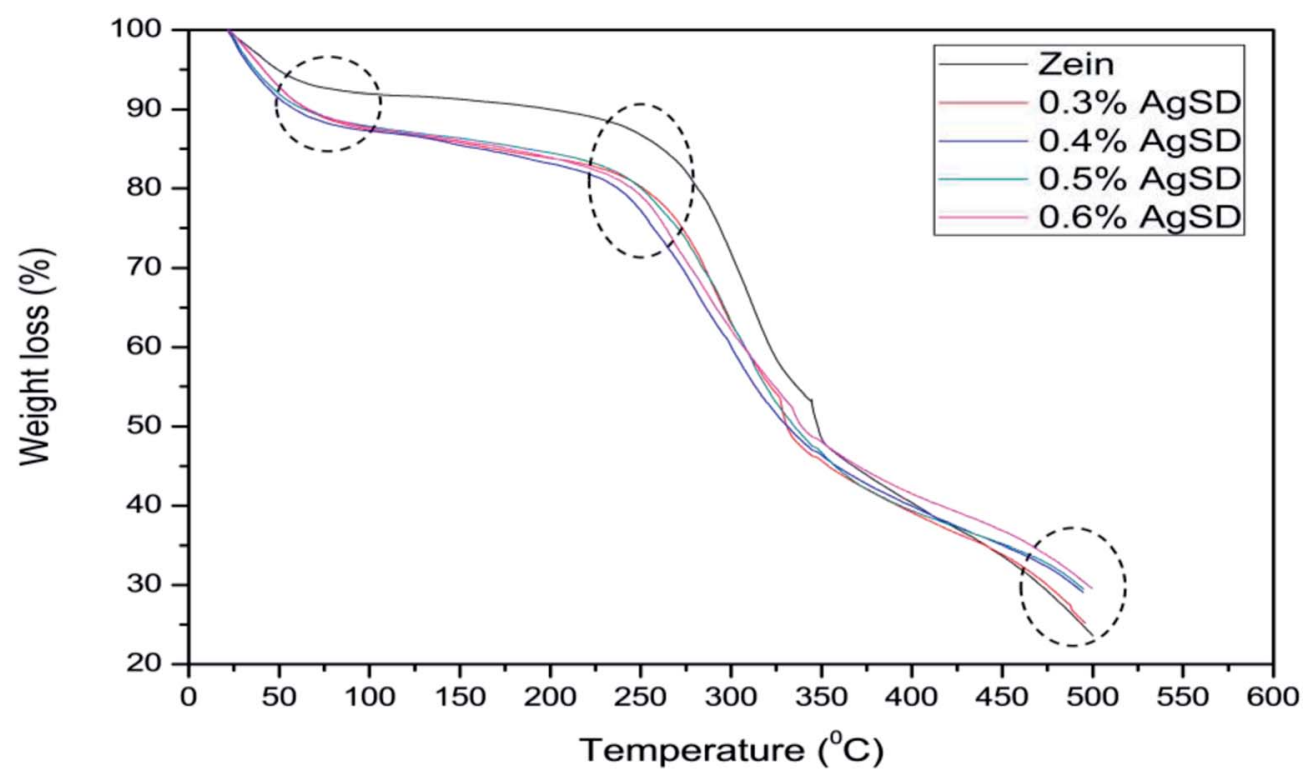

Fig. 7 Thermogravimetric analysis of zein and AgSD/zein nanofiber mats. 


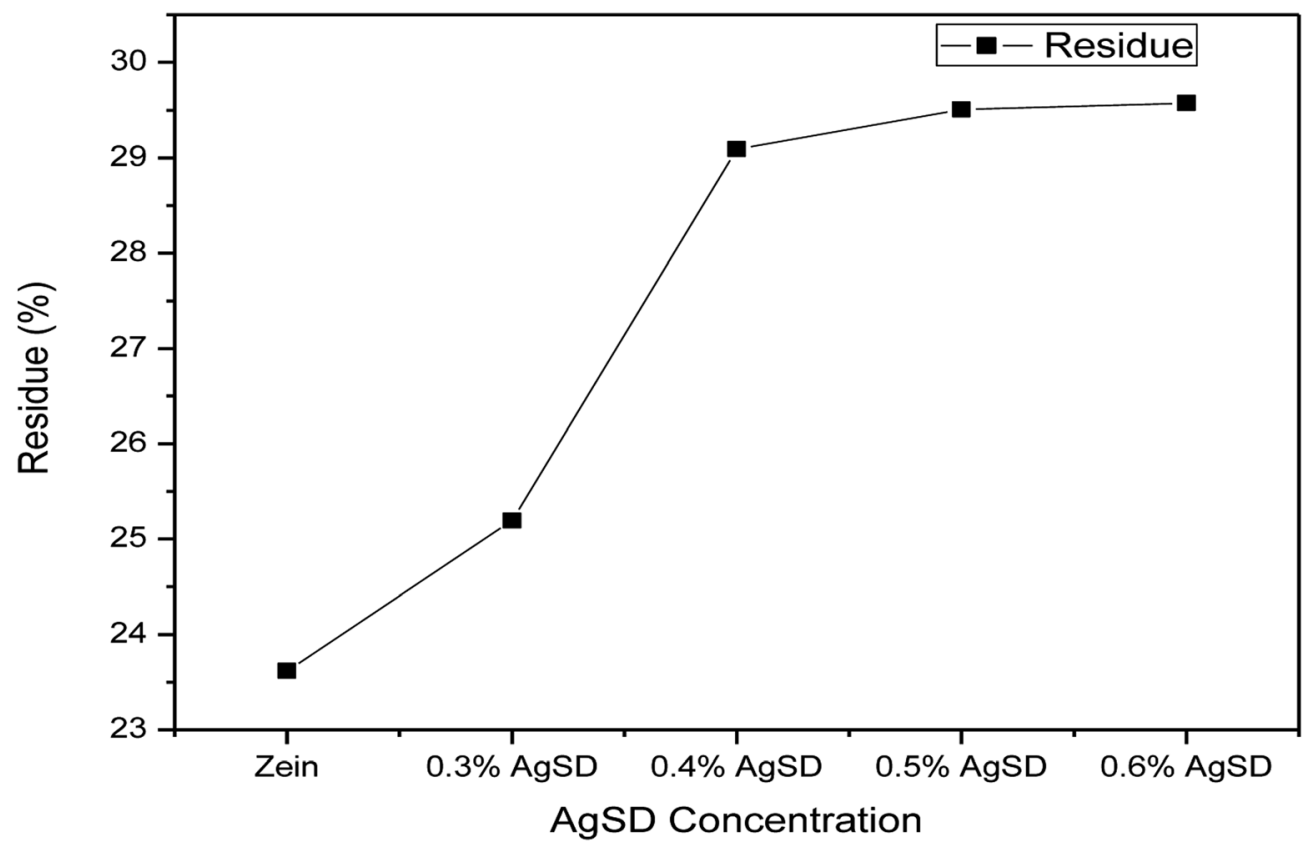

Fig. 8 Comparison of residue of zein and AgSD/zein nanofibers.

\section{Thermogravimetric analysis (TGA)}

Thermogravimetric analysis was done to analyze the effect of AgSD on thermal degradation of zein. TGA graph is divided in three parts. Where $1^{\text {st }}$ part (up to $100{ }^{\circ} \mathrm{C}$ ) shows removal of moisture, $2^{\text {nd }}$ part shows the thermal degradation onset and the last part shows combustion zone where we analyze the residual amount. It can be shown in Fig. 7 that $1^{\text {st }}$ part of graph showed higher moisture absorption in zein/AgSD composite nanofibers mats as graph went downward because of removal of moisture contents in nanofiber mats. $2^{\text {nd }}$ part of graph, which declares the thermal degradation onset, showed that onset of pure zein nanofibers was at $270{ }^{\circ} \mathrm{C}$ and that was for zein/AgSD nanofibers

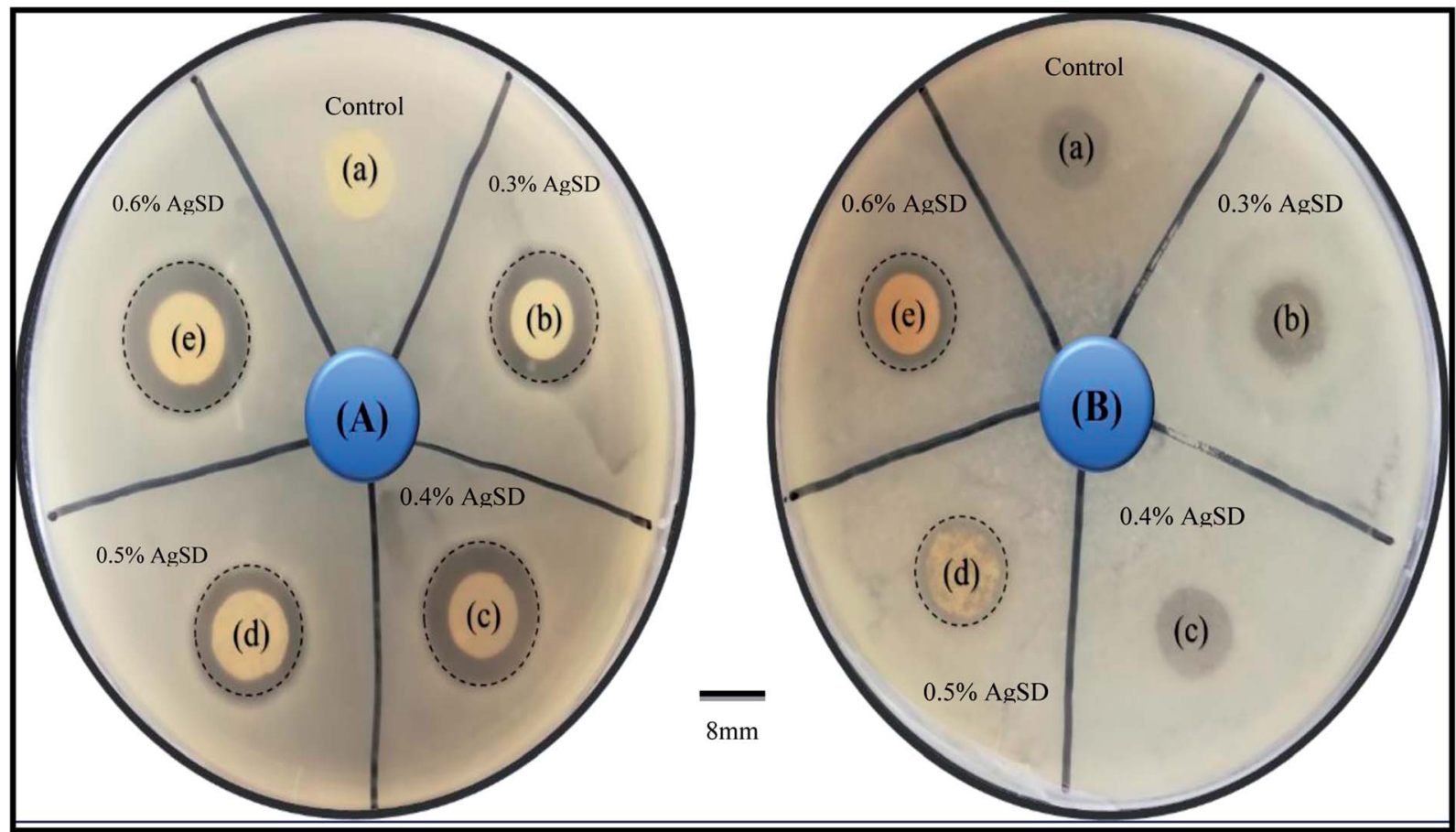

Fig. 9 Antibacterial activity test by disk diffusion method (A) E. coli (B) Bacillus ((a) control, (b) $0.3 \%$ AgSD in zein, (c) $0.4 \%$ AgSD in zein, (d) $0.5 \%$ AgSD in zein, (e) $0.6 \%$ AgSD in zein). 


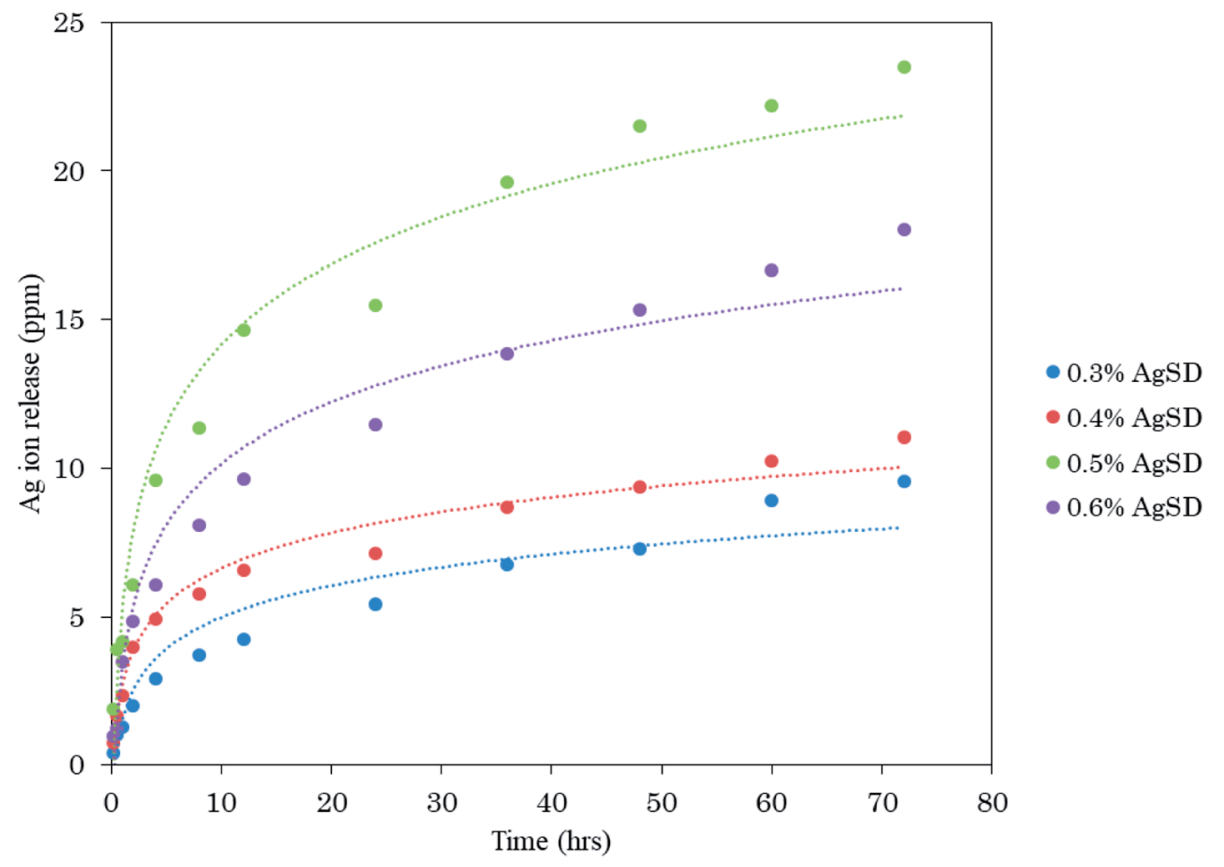

Fig. 10 Investigation of release properties of AgSD loaded zein nanofibers.

started from $260^{\circ} \mathrm{C}$ which showed slight lower thermal stability by addition of AgSD to pure zein but not much affected. Offset of thermal degradation curve for both pure zein and AgSD added samples was found to be at same temperatures. Last part of TGA graph showed different residual percentage for each sample. As concentration of AgSD increased in zein, the residue was also increased which clearly presented the presence of AgSD in zein nanofibers.

Fig. 8 shows broad view of residual amount of zein nanofibers and zein/AgSD nanofibers. Pure zein nanofibers had $23.31 \%$ residue content at $500{ }^{\circ} \mathrm{C}$, residue contents for $0.3 \%$ $\mathrm{AgSD}, 0.4 \% \mathrm{AgSD}, 0.5 \% \mathrm{AgSD}$, and 0.6\% AgSD were 25.16\%, $29.089 \%, 29.50 \%$, and $29.58 \%$ respectively. So, it was concluded that addition of AgSD in zein caused increase in residual contents of nanofibers.

\section{Anti-bacterial activity test}

In order to investigate of antibacterial properties, disc diffusion method was carried out as shown in Fig. 9.

Bacillus and E. coli bacteria strain were used as Gram-positive and Gram-negative respectively. The antibacterial effectiveness of AgSD released from zein nanofibers was determined from the zone inhibition of the bacteria. The antibacterial activity of zein nanofibers loaded by drug observed with both strains of bacteria in comparison to control. Excellent antibacterial efficacy was attributed to sample with $0.6 \% \mathrm{AgSD}$. It was observed that increasing the amount of drug had a significant effect on the antibacterial activity of nanofibers mat. For example, some of the samples were degraded by bacterial action due to insufficient amount of drug in those samples (Fig. 1 B). Herein, from the results it was concluded that, the sample with a drug concentration of $0.6 \%$ showed the excellent antibacterial activity in comparison to control and other samples. Where, samples (a), (b), (c), (d), (e) represent pure zein, 0.3\% AgSD, $0.4 \% \mathrm{AgSD}, 0.5 \% \mathrm{AgSD}$, and $0.6 \% \mathrm{AgSD}$ respectively.

\section{Investigation of release properties}

In order to analyze release behavior of $\mathrm{Ag}$ from zein nanofibers. $0.5 \mathrm{~g}$ of AgSD/zein nanofibers (each sample with different AgSD concentration) was immersed in $50 \mathrm{ml}$ of deionized water and keep on stirring (slowly) for 72 hours and analyzed by ICP. It was observed (Fig. 10) that release of $\mathrm{Ag}$ was gradually increased with increasing concentration of AgSD in zein nanofibers. It was also observed that samples were not dissolved in deionized water over 72 hours. Sample with $0.6 \%$ AgSD concentration showed a maximum of $23.5 \mathrm{ppm}$ release of Ag ion. Samples with $0.3 \%, 0.4 \%$, and $0.5 \% \mathrm{AgSD}$ concentration showed $9.54 \mathrm{ppm}$, $11.01 \mathrm{ppm}$, and $18.05 \mathrm{ppm}$ of $\mathrm{Ag}$ ion. From release test, it was observed that prepared nanofiber mats has good release properties and can be effective for wound care applications.

\section{Conclusions}

AgSD loaded zein nanofibers were successfully prepared by above mentioned methods. From antibacterial activity test it was concluded that prepared nanofiber mats will be effective in wound care applications. Nanofibers with $0.6 \% \mathrm{AgSD}$ in zein, had shown excellent antibacterial activity for both Gram positive as well as Gram negative bacteria, so from this result we recommend $0.6 \%$ concentration of drug for further applications. Release test was carried out by ICP which also showed excellent release properties of sample having $0.6 \%$ concentration of AgSD as compared to other samples with low concentration of AgSD. SEM images had shown uniform morphology 
with no beads formation at $25 \%$ of zein concentration. After analysis of XPS and EDX, it was concluded that AgSD did not disturbed the crystalline structure of zein and was present in the nanofibers. FTIR study had shown that there was no chemical reaction involved between zein and AgSD. Thermal properties of AgSD/zein nanofibers were also analyzed which declared that nanofibers containing AgSD were also stable above $260{ }^{\circ} \mathrm{C}$. The degradation temperature was not increased by the addition of AgSD which was sign that degradability of zein was not much disturbed due to presence of AgSD in nanofibers. Finally, from all above mentioned results and discussions it was concluded that AgSD loaded zein nanofibers have great potential to be used as wound dressing, and will be applicable on industrial scale manufacturing of nonwoven wound dressings.

\section{Conflicts of interest}

There are no conflicts to declare.

\section{References}

1 D. Kharaghani, Y. Kee Jo, M. Q. Khan, Y. Jeong, H. J. Cha and I. S. Kim, Electrospun antibacterial polyacrylonitrile nanofiber membranes functionalized with silver nanoparticles by a facile wetting method, Eur. Polym. J., 2018, 108, 69-75, DOI: 10.1016/j.eurpolymj.2018.08.021.

2 H. Lee, G. Xu, D. Kharaghani, et al., Electrospun tri-layered zein/PVP-GO/zein nanofiber mats for providing biphasic drug release profiles, Int. J. Pharm., 2017, 531(1), 101-107, DOI: 10.1016/j.ijpharm.2017.08.081.

3 M. Q. Khan, D. Kharaghani, N. Nishat, et al., The development of nanofiber tubes based on nanocomposites of polyvinylpyrrolidone incorporated gold nanoparticles as scaffolds for neuroscience application in axons, Text. Res. J., 2018, 004051751880118, DOI: 10.1177/0040517518801185.

4 U. Dashdorj, M. K. Reyes, A. R. Unnithan, et al., Fabrication and characterization of electrospun zein/Ag nanocomposite mats for wound dressing applications, Int. J. Biol. Macromol., 2015, 80, 1-7, DOI: 10.1016/ j.ijbiomac.2015.06.026.

5 C. L. Fox and S. M. Modak, Mechanism of Silver Sulfadiazine Action on Burn Wound Infections Mechanism of Silver Sulfadiazine Action on Burn Wound Infections, Antimicrob. Agents Chemother., 1974, 5(6), 582-588, DOI: 10.1128/ AAC.5.6.582.

6 I. Alan Holder, In vitro inactivation of silver sulphadiazine by the addition of cerium salts, Burns, 1982, 8(4), 274-277, DOI: 10.1016/0305-4179(82)90009-2.

7 M. Rai, A. Yadav and A. Gade, Silver nanoparticles as a new generation of antimicrobials, Biotechnol. Adv., 2009, 27(1), 76-83, DOI: 10.1016/j.biotechadv.2008.09.002.

8 L. Francis, F. Giunco, A. Balakrishnan and E. Marsano, Synthesis, characterization and mechanical properties of nylon-silver composite nanofibers prepared by electrospinning, Curr. Appl. Phys., 2010, 10(4), 1005-1008, DOI: 10.1016/j.cap.2009.12.025.
9 A. T. Hang, B. Tae and J. S. Park, Non-woven mats of poly(vinyl alcohol)/chitosan blends containing silver nanoparticles: fabrication and characterization, Carbohydr. Polym., 2010, 82(2), 472-479, DOI: 10.1016/ j.carbpol.2010.05.016.

10 B. Pant, H. R. Pant, D. R. Pandeya, et al., Characterization and antibacterial properties of $\mathrm{Ag}$ NPs loaded nylon-6 nanocomposite prepared by one-step electrospinning process, Colloids Surf., A, 2012, 395, 94-99, DOI: 10.1016/ j.colsurfa.2011.12.011.

11 A. GhavamiNejad, A. Rajan Unnithan, A. Ramachandra Kurup Sasikala, et al., Mussel-Inspired Electrospun Nanofibers Functionalized with Size-Controlled Silver Nanoparticles for Wound Dressing Application, ACS Appl. Mater. Interfaces, 2015, 7(22), 12176-12183, DOI: 10.1021/ acsami.5b02542.

12 C. H. Yang, L. S. Wang, S. Y. Chen, et al., Microfluidic assisted synthesis of silver nanoparticle-chitosan composite microparticles for antibacterial applications, Int. J. Pharm., 2016, 510(2), 493-500, DOI: 10.1016/ j.ijpharm.2016.01.010.

13 B. Maharjan, M. K. Joshi, A. P. Tiwari, C. H. Park and C. S. Kim, In-situ synthesis of AgNPs in the natural/ synthetic hybrid nanofibrous scaffolds: Fabrication, characterization and antimicrobial activities, J. Mech. Behav. Biomed. Mater., 2017, 65, 66-76, DOI: 10.1016/ j.jmbbm.2016.07.034.

14 T. Miyoshi, K. Toyohara and H. Minematsu, Preparation of ultrafine fibrous zein membranes via electrospinning, Polym. Int., 2005, 54(8), 1187-1190, DOI: 10.1002/pi.1829.

15 S. Torres-Giner, E. Gimenez and J. M. Lagaron, Characterization of the morphology and thermal properties of zein prolamine nanostructures obtained by electrospinning, Food Hydrocolloids, 2008, 22(4), 601-614, DOI: 10.1016/j.foodhyd.2007.02.005.

16 B. Zhang, Y. Luo and Q. Wang, Development of silver-zein Composites as a promising antimicrobial agent, 2010:pp. 2366-2375.

17 F. Kayaci and T. Uyar, Electrospun zein nanofibers incorporating cyclodextrins, Carbohydr. Polym., 2012, 90(1), 558-568, DOI: 10.1016/j.carbpol.2012.05.078.

18 K. Karthikeyan, S. Guhathakarta, R. Rajaram and P. S. Korrapati, Electrospun zein/eudragit nanofibers based dual drug delivery system for the simultaneous delivery of aceclofenac and pantoprazole, Int. J. Pharm., 2012, 438(12), 117-122, DOI: 10.1016/j.ijpharm.2012.07.075.

19 Y. N. Jiang, H. Y. Mo and D. G. Yu, Electrospun drug-loaded core-sheath $\mathrm{PVP} /$ zein nanofibers for biphasic drug release, Int. J. Pharm., 2012, 438(1-2), 232-239, DOI: 10.1016/ j.ijpharm.2012.08.053.

20 L. Y. Huang, C. Branford-White, X. X. Shen, D. G. Yu and L. M. Zhu, Time-engineeringed biphasic drug release by electrospun nanofiber meshes, Int. J. Pharm., 2012, 436(12), 88-96, DOI: 10.1016/j.ijpharm.2012.06.058.

21 J. M. Yang, Z. L. sheng, D. G. Yu and J. Liu, Coaxial electrospinning with acetic acid for preparing ferulic acid/ zein composite fibers with improved drug release profiles, 
Colloids Surf., B, 2013, 102, 737-743, DOI: 10.1016/ j.colsurfb.2012.09.039.

22 W. Huang, T. Zou, S. Li, J. Jing, X. Xia and X. Liu, DrugLoaded Zein Nanofibers Prepared Using a Modified Coaxial Electrospinning Process, AAPS PharmSciTech, 2013, 14(2), 675-681, DOI: 10.1208/s12249-013-9953-1.

23 R. Paliwal and S. Palakurthi, Zein in controlled drug delivery and tissue engineering, J. Controlled Release, 2014, 189, 108122, DOI: 10.1016/j.jconrel.2014.06.036.

24 A. R. Unnithan, G. Gnanasekaran, Y. Sathishkumar, Y. S. Lee and C. S. Kim, Electrospun antibacterial polyurethanecellulose acetate-zein composite mats for wound dressing, Carbohydr. Polym., 2014, 102(1), 884-892, DOI: 10.1016/ j.carbpol.2013.10.070.
$25 \mathrm{X} . \mathrm{Hu}$, S. Liu, G. Zhou, Y. Huang, Z. Xie and X. Jing, Electrospinning of polymeric nanofibers for drug delivery applications, J. Controlled Release, 2014, 185(1), 12-21, DOI: 10.1016/j.jconrel.2014.04.018.

26 S. López-Alcaide, M. Nakamura, R. Macip-Ríos and E. Martínez-Meyer, Does behavioural thermoregulation help pregnant Sceloporus adleri lizards in dealing with fast environmental temperature rise?, J. Herpetol., 2014, 24(1), 41-47, DOI: 10.1097/00042752-200605000-00013.

27 E. Corradini, P. S. Curti, A. B. Meniqueti, A. F. Martins, A. F. Rubira and E. C. Muniz, Recent advances in foodpacking, pharmaceutical and biomedical applications of zein and zein-based materials, Int. J. Mol. Sci., 2014, 15(12), 22438-22470, DOI: 10.3390/ijms151222438. 